\title{
Evaluación del proceso de desencalado de residuos de descarnes de un proceso de curtición
}

\section{Evaluation of the process of descarnes deliming waste of a process of tanning}

\author{
Peñates F Sebastian ${ }^{1}$, Guzmán D Natalia ${ }^{1}$ Ing, Aguas M Yelitza ${ }^{1 *}$ M.Sc., \\ Martínez M Ana ${ }^{1}$ Esp, Cury R Katia ${ }^{1}$ MSc.
}

\section{Keywords:}

Deliming; tanning; waste; tannery.
Palabras Clave:

Desencalado; curtición; residuos; curtiembre

\section{Abstract}

The productive transformation process in furs leather developed in the tanning industry generates different types of waste that should have a proper handling. This research consisted of find a separation of waste lime treatment of suede desencalado of the tanning process stage the process of tanning to be used in different applications. Descarnes whitewashed were selected for the implementation of 6 treatments with three replications of deliming through baths with water at room temperature by controlling the factors time in 3 levels (2, 3 and 4 hours) and sulfuric acid as an agent decalcifier at two levels 1 and $2(\mathrm{~N})$. The best results in this process were obtained with the treatment with a concentration of sulfuric acid $2 \mathrm{~N}$ and an agitation time of 3 hours.

\section{Resumen}

El proceso productivo de transformación de las pieles en cuero desarrollado en la industria curtiente genera diferentes tipos de residuos que deben tener un adecuado manejo. Esta investigación consistió en buscar un tratamiento de separación de cal de los residuos de descarne de la etapa de desencalado del proceso de curtición que le permitan ser aprovechados en diferentes usos. Se seleccionaron descarnes encalados, para la aplicación de 6 tratamientos con tres repeticiones de desencalado mediante baños con agua a temperatura ambiente controlando los factores tiempo en 3 niveles (2,3 y 4 Horas) y ácido sulfúrico como agente desencalante en dos niveles ( 1 y $2 \mathrm{~N}$ ). Los mejores resultados en este proceso se obtuvieron con el tratamiento con una concentración de ácido sulfúrico al $2 \mathrm{~N}$ y un tiempo de agitación de 3 horas. 


\section{Introducción}

En la actualidad, existe un alto grado de interés y preocupación por el cuidado del medio ambiente. Es por esto que todas las actividades llevadas a cabo por cualquier tipo de industria u organización deben ser realizadas en armonía con el medio ambiente, de tal forma que los impactos ocasionados en este disminuyan con el transcurrir del tiempo. La industria de las curtiembres no omite esta tendencia, por lo que los aspectos ambientales son considerados con mayor frecuencia, con el fin de alcanzar una mejora en la productividad y eficiencia del proceso. Teniendo en cuenta que las actividades llevadas a cabo en el proceso productivo de las curtiembres están estrechamente vinculadas con el ambiente, se hace necesario que este sector busque minimizar el impacto negativo que causa en el medio ambiente, independientemente del tamaño y localización de la industria.

En Colombia las actividades del sector curtiembres inician alrededor de los años veinte en los departamentos de Antioquia y de los años cincuenta en Cundinamarca y posteriormente en Nariño, Quindío, Risaralda, Atlántico, Valle del Cauca, Tolima, Bolívar, Santander y Huila (CNPML 2004), representadas en su mayor porcentaje por pequeñas y medianas empresas que generan empleos directos e indirectos para la población donde se encuentran ubicadas. En 2004, el Centro Nacional de Producción Más Limpia y Tecnologías Ambientales, CNPMLTA, realizó un diagnóstico sobre el estado del sector en el país, donde se concluye que está compuesto por un $77 \%$ de microempresas, $19 \%$ de pequeñas industrias, un $3 \%$ por medianas y un $1 \%$ de gran industria; en ese año se registraron en el país cerca de 671 curtiembres, con producción de 271.000 pieles/ mes (ALZATE, 2008), sin embargo la mayoría de empresas cuenta con maquinaria obsoleta y niveles incipientes de tecnología, no realizan inversión en infraestructura adecuada y a esto le sumamos que muchas de ellas tienen operarios con baja capacitación (CNPML, 2004).

El proceso de curtido de pieles se puede realizar de dos formas que son: curtido al cromo y curtido al vegetal, la primera se utiliza para elaborar cueros de textura suave y calibre delgado para aplicación en el calzado y marroquinería, mediante la utilización de cromo, sales metálicas, formaldehidos (CORREDOR, 2006), con el fin de obtener un cuero de mejor calidad que permita un mejor manejo al momento de su manufactura. Esta forma de curtición es considerada la más contaminante en relación a la otra forma de curtido que se hace al vegetal, a través de la utilización de taninos, la cual produce un cuero más grueso, resistente y poco flexible, utilizado también la industria del calzado. De esta manera por cada $1000 \mathrm{Kg}$ de piel salada que entra al proceso se obtienen de producto $150 \mathrm{Kg}$ de cuero acabado, $40 \mathrm{Kg}$ de solventes son emitidos a la atmosfera, y $850 \mathrm{Kg}$ de residuos sólidos son producidos (AGUAS et al., 2016), así mismo se requieren $450 \mathrm{Kg}$ de insumos químicos; dentro de los que se destaca principalmente el cromo que al estar presente en los cuerpos de agua como $\mathrm{Cr}+3$, poco soluble y muy estable (elemento traza esencial), y como $\mathrm{Cr}+6$, menos estable pero más soluble, con una alta movilidad entre sustratos, siendo mutagénico y carcinogénico en humanos, puede alterar seriamente el equilibrio biológico causando efectos tóxicos, ya que es rápidamente absorbido por las membranas biológicas (TÉLLEZ et al. 2004, LOAYZA 2006, MANCERA-RODRÍGUEZ y ÁLVAREZ 2006).

Básicamente el proceso de curtición de pieles consta de 3 fases, la primera llamada etapa de ribera, la cual consiste en recepcionar la materia prima y eliminar la grasa, carne y pelo, hasta el dividido de las pieles, produciéndose grandes cantidades de residuos sólidos como tierra, sangre, pelo, estiércol, grasa, descarnes, sal entre otros, y cuyos residuos líquidos presentan altos niveles de alcalinidad, sulfuro, nitrógenos, solidos disueltos y suspendidos, grasas y aceites, así como altas demandas de DBO5 Y DQO. En esta fase se produce el $70 \%$ de la contaminación total generada por una curtiembre (ANDRIOLI et al., 2015). La siguiente etapa se denomina etapa de curtido, y es en esta donde se convierte la piel en cuero, ya sea mediante el usos de sales de cromo y otras sustancias, que generan efluentes líquidos, baja demanda de $\mathrm{DBO}_{5}$ y $\mathrm{DQO}$ y altos contenidos de sales y ácidos, además de algunos residuos sólidos como pelo, bordes, carne y grasa; o mediante el uso de taninos provenientes de ciertos vegetales como el castaño, la mimosa, el dividivi, los cuales poseen altos contenidos de 
componentes tánicos orgánicos, y que producen amoniaco, desechos alcalinos, ácidos y agua altamente coloreada (CORREDOR, 2006).

La última etapa del proceso de curtición, denominada terminado o acabado, donde finalmente las pieles se tiñen y engrasan, se sujetan con estacas $u$ otros soportes para mantenerlas estiradas y se lavan para su entrega al consumidor. Se presentan altas descargas de colorantes y grasas.

El municipio de Sampuès, ubicado en el departamento de Sucre, Colombia, cuenta con medianas empresas curtidoras de pieles, que utilizan el proceso de curtido al vegetal, con el dividivi como agente curtiente. En este proceso de curtición semiartesanal se inicia con la recepción de la piel, donde se extrae parte de residuos adheridos a esta, en la etapa de apelambrado la piel se somete a encalado o pelambre sumergiéndola en una lechada de $\mathrm{Ca}(\mathrm{OH})_{2}$ por un tiempo suficiente para conseguir el desprendimiento de pelos; posteriormente se retiran residuos de carne y sebos mediante el descarnado; la piel libre de pelos y descarnes es desencalada mediante baños de agua, para pasar a curtición con dividivi previamente molido, lavar y secar al sol. Resultado de este proceso es la generación de cantidades considerables de residuos líquidos y sólidos, estos últimos residuos corresponden a residuos de descarne, $5600 \mathrm{~kg}$ por mes, derivados del procesamiento de 800 pieles; también se obtienen, pelos y restos de dividivi no absorbido y lodos de cal (Información primaria. Operarios del proceso de descarnado Curtiembres de Sampués, 2015). Los descarnes como son separados antes de someter las pieles a curtición, no reciben las sustancias curtientes que estabilizan las propiedades y evitan cambios naturales de descomposición y putrefacción, sin embargo salen con altos contenidos de cal adheridos, lo que dificulta su aprovechamiento puesto que este tipo de residuos tienen altos contenidos de grasa y proteínas.

Los descarnes representan entre un $20-35 \%$ del peso inicial de las pieles (VICENTE, 2010). No obstante estos residuos sólidos pueden generan efectos negativos sobre el medio ambiente, como malos olores, emisión de sulfuros, amoniaco y otros solventes; proliferación de insectos, roedores y aves de rapiña, por ser un material putrescible; y que provocan enfermedades de tipo respiratorio, intestinales y dermatológicas (MARTINEZ Y PARIS, 2010). En Colombia, se utilizan como abono en la fertilización de pastizales que tardan un año en asimilarlo. Sin embargo, también se depositan en botaderos ilegales a cielo abierto localizados en sitios rurales (VICENTE, 2010). Además, las carnazas se emplean como materia prima para gelatinas. Estos residuos están formados de tejido adiposo, conjuntivo y muscular que pueden ser aprovechados en el sector cosmético, para elaborar concentrados para animales, jabones, gelatinas, para el mismo tratamiento del cuero porque se usa junto con los aceites en el engrase, entre otros usos. Dejan de ser un desecho de las curtiembres para convertirse en una fuente de materia prima (GORDILLO Y TOLEDO, 2014).

Para poder aprovechar estos residuos de descarnes es necesario retirar el exceso de cal adherido a este material, proceso que se denomina desencalado. Es así como el objetivo de esta investigación fue buscar un tratamiento de separación de cal de los residuos de descarne de la etapa de desencalado del proceso de curtición desarrollado en Sampuès-Sucre Colombia que permita dejarlos en condiciones óptimas para ser aprovechados en diferentes usos.

\section{Materiales y métodos}

La investigación se desarrolló en las instalaciones del laboratorio Conservación del recurso hídrico y alimentos, de la Universidad de Sucre, campus Puerta Roja, en Sincelejo, Sucre.

Se recolectaron residuos de descarnes derivados del proceso de elaboración del cuero, provenientes de cuatro células de producción de la Curtiembre ubicada en el municipio de Sampués, localizado a $19 \mathrm{Km}$ de Sincelejo, departamento de Sucre, Colombia; se tomaron muestras homogéneas, las cuales se acondicionaron se dividieron hasta obtener una superficie aproximada de $2 \mathrm{~cm}^{2}$, para mejorar su manejo. Se conservaron en refrigeración a una temperatura máxima de $5^{\circ} \mathrm{C}$ hasta su uso. 
A las muestras se les evaluaron los parámetros fisicoquímicos de: $\mathrm{pH}$, se determinó con un pHmetro de mesa modelo UB-10, marca Denver Instrument, para medir el $\mathrm{pH}$ presente en cada una de las muestras según A.O.A.C. 981.12/90, previamente calibrado con soluciones buffer de $\mathrm{pH} 4$ y 7; contenido de grasa mediante equipo Soxhlet según la A.O.A.C 24.005/95, la presencia de cal se determinó empleando fenolftaleína como indicador de $\mathrm{pH}$, adicionando una gota del indicador sobre un corte de carnaza, antes y después del desencalado; la presencia del color rosado indicó un $\mathrm{pH}$ superior a 8,2 evidenciando que el desencalado realizado no fue efectivo. Si se torna incoloro indica un $\mathrm{pH}$ inferior a 8,2 significando un desencalado efectivo (ARANGO et al., 2015; AGUAS et al., 2017). Para evaluar la presencia de cal se estableció una escala de medición, donde se utilizó el número 0 como un indicador de su carencia, y el número 1 como un valor que representó su presencia.

Los tratamientos de desencalado de los residuos de descarne se realizaron en recipientes con agua a temperatura ambiente $\left(28^{\circ} \mathrm{C}\right)$ con agitación constante y utilizando agitadores eléctricos, modelo ER 10, que simularon los baños realizados en los bombos de madera que utilizan en la curtiembre, adicionándoles ácido sulfúrico $\mathrm{H}_{2} \mathrm{SO}_{4}$ llevando las muestras de descarne hasta un rango de $\mathrm{pH}$ que osciló entre 5.5 y 7 . Se desarrolló un sistema multifactorial de $2 \times 3$, siendo los factores: ácido sulfúrico como agente desencalante en dos niveles $(1$ y $2 \mathrm{~N})$ y tiempo de agitación en tres niveles (2, 3 y 4 horas). Desarrollándose seis tratamientos con tres repeticiones cada uno, para un total de 18 unidades experimentales.

Se compararon los resultados obtenidos de las muestras antes y después de los tratamientos aplicados y se analizaron los datos empleando el paquete estadístico statgraphics centurium; observándose que los resultados obtenidos presentaron diferencias estadísticas significativas $(P \leq 0,05)$. Posteriormente se utilizó la prueba de rangos múltiples con un nivel de confiabilidad del $95 \%$, para determinar cuáles medias son diferentes de otras y finalmente seleccionar el mejor tratamiento.

\section{Resultados y discusión}

Los residuos de descarne frescos presentaron un $\mathrm{pH}$ básico entre $9.5-11.5$, mientras que el contenido de grasa fue aproximadamente de $63.5 \%$. Luego de aplicar los tratamientos, los residuos de descarne desencalados presentaron variación en el porcentaje de grasa entre $50-57 \%$ como se muestra en la figura 1.

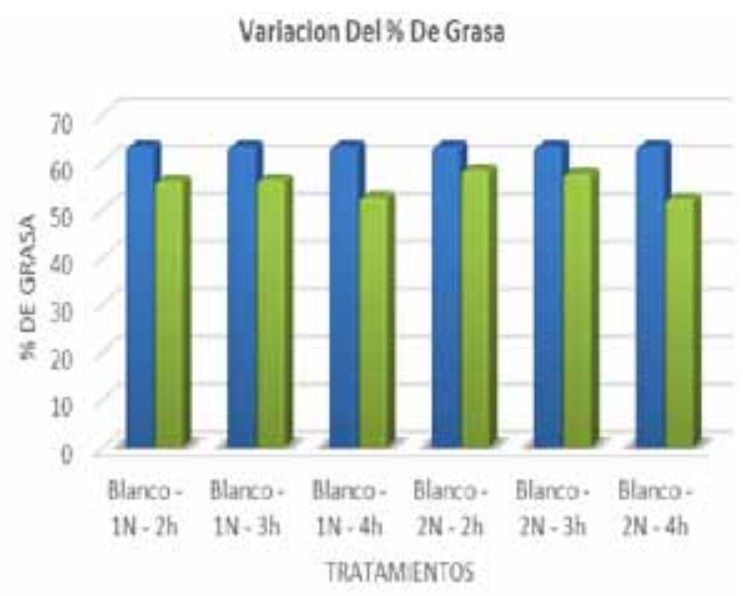

Figura1. Variación del \% de grasa

El análisis de varianza del diseño experimental (Tabla 1), demostró que existe un efecto significativo por parte de uno de los dos factores analizados. Dicho factor fue la concentración del agente desencalante, el cual tuvo una incidencia significativa, por lo que se evidencio pérdidas en el porcentaje de grasa de los residuos de descarne. La prueba de múltiples rangos (Tabla 2) mostró que no existen diferencias entre las concentraciones de $1 \mathrm{~N}-2 \mathrm{~N}$ y Blanco $-1 \mathrm{~N}$. Sin embargo, en la comparación de Blanco - $2 \mathrm{~N}$ si se evidenciaron efectos significativos sobre el porcentaje grasa, lo cual se debe a que al estar más concentrado

Tabla 1. Análisis de varianza del diseño experimental

\begin{tabular}{lccccc}
\hline Fuente & SC & GI & CM & Rf & Vp \\
Efectos principales & & & & & \\
A:Concentracion & 190.417 & 2 & 95.2087 & 8.91 & 0.0025 \\
B:Tiempo & 5.87866 & 2 & 2.93933 & 0.27 & 0.7631 \\
$\begin{array}{l}\text { Interacciones } \\
\text { Residuos }\end{array}$ & 171.039 & 16 & 10.6899 & \\
\hline TOTAL & 367.335 & 20 & & & \\
\hline sc: Suma de Cuadrados; CM: Cuadrado Medio; Rf: Razón-F; Vp: Valor-P
\end{tabular}

SC: Suma de Cuadrados; CM: Cuadrado Medio; Rf: Razón-F; Vp: Valor-P 
Tabla 2: Prueba de rangos múltiples

\begin{tabular}{|c|c|c|c|c|c|c|c|c|}
\hline Concentración & Casos & Media LS & Sigma LS & $\begin{array}{c}\text { Grupos } \\
\text { Homogéneos }\end{array}$ & Contraste & Sig. & Diferencia & +/- Límites \\
\hline $2 \mathrm{~N}$ & 9 & 54.2018 & 1.08985 & $x$ & $1 N-2 N$ & & 2.74967 & 4.63669 \\
\hline $1 \mathrm{~N}$ & 9 & 56.9514 & 1.08985 & $x x$ & $1 \mathrm{~N}$ - Blanco & & -6.42389 & 6.55728 \\
\hline Blanco & 3 & 63.3753 & 1.88767 & $x$ & $2 \mathrm{~N}-$ Blanco & * & -9.17356 & 6.55728 \\
\hline
\end{tabular}

*grados de libertad

el ácido sulfúrico facilita la ruptura de la envoltura de las proteínas y fosfolípidos, por lo que el ácido digiere rápidamente los complejos de proteína y carbohidratos, logrando así que se libere la grasa (MARTíNEZ Y PARIS, 2010). No obstante se estos residuos presentan cantidades de grasa interesantes para la fabricación de diferentes subproductos como jabones entre otros. El matadero San Jorge ubicado en Cochabamba Bolivia, desde 1992 viene desarrollando procesos de extracción de grasa a partir de residuos de matadero, para la producción de jabón. Trayendo consigo beneficios ambientales y económicos para la región y la empresa. En el primer caso se evita la acumulación de materia grasa en el relleno sanitario y en el segundo se crea una nueva fuente de ingreso económico para la empresa (VICENTE, F. 2010).

Para el caso del pH se observó una disminución de su valor en las muestras de descarne después de aplicar los tratamientos, se mantuvieron en un rango entre 5,5 y 7 (Figura 2), por debajo del valor referencia del método utilizado $(\mathrm{pH}<8,2)$. Esto implica que se favoreció la eliminación de cal de los descarnes dado que el pH juega un

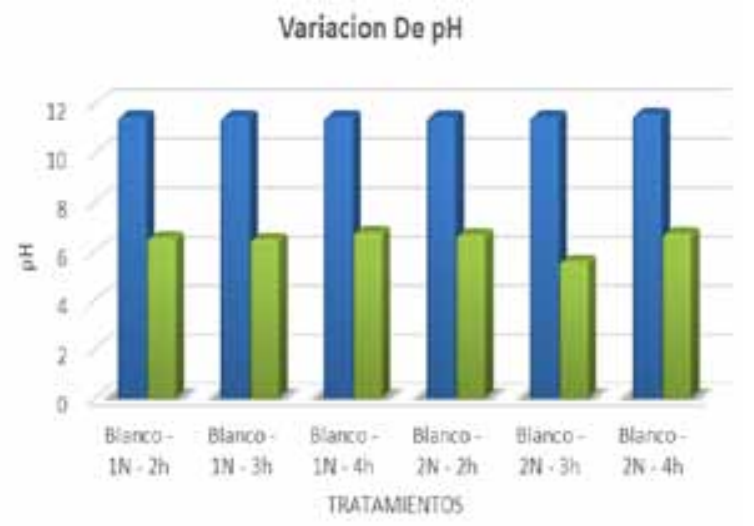

Figura 2. Variación De $\mathrm{pH}$ papel influyente en el proceso de desencalado, ya que indica si este fue efectivo o no (AGUAS, et al., 2016). De igual manera, el descenso del pH indica que gran parte de la cal estaba impregnada capilarmente y superficialmente reaccionó con el ácido sulfúrico para formar una sal de mayor solubilidad conocida como sulfato de calcio que tiene un $\mathrm{pH}$ de 6.98, y que por sus características es más fácil separarla de las muestra de carnaza. Esta sal formada no es toxica y sirve como nutriente o suplemento dietario en drogas y especialmente en comidas para animales (SILVA Y GONZÁLEZ, 1993).

Por último, se evaluó la presencia de cal de una manera cualitativa, obteniendo resultados positivos (1) que alertaban la presencia de cal en 4 de los 6 tratamientos. Estos fueron: $1 \mathrm{~N}$ $2 \mathrm{~h}, 1 \mathrm{~N}-3 \mathrm{~h}, 1 \mathrm{~N}-4 \mathrm{~h}$ y $2 \mathrm{~N}-2 \mathrm{~h}$. De acuerdo a lo anterior, los tratamientos que tuvieron un resultado satisfactorio, es decir, sin presencia de cal (0) fueron $2 \mathrm{~N}-3 \mathrm{~h}$ y $2 \mathrm{~N}-4 \mathrm{~h}$. Lo anterior corrobora los resultados obtenidos por AGUAS et al.; 2017 donde se estableció que el desencalado por medio de baños termotratados tipo bombo es una técnica capaz de retirar la cal hidratada y mantener un elevado contenido de grasa en los residuos de descarne de una curtiembre.

Teniendo en cuenta que la curtiembre objeto de estudio ubicada en el municipio de Sampuès, no aprovecha este tipo de residuos sino que simplemente los depositan en un relleno sanitario, se hace necesario buscar alternativas que ayuden a mitigar el impacto ambiental negativo que se está produciendo y al mismo tiempo buscar valorizar o comercializar estos residuos como subproductos para otras industrias, por lo que esta investigación puede ser tomada como punto de partida para este fin. 
De igual manera cabe resaltar investigaciones como la realizada por la facultad de ciencias agropecuarias de la universidad de Camagüey (Cuba) donde se realizó la caracterización de los residuos de descarnes y carnaza revisando sus potencialidades como fuente energético-proteica en la nutrición de los monogástricos. Concluyendo que la carnaza debido a sus potencialidades energéticas proteicas, a su porcentaje de grasa y cenizas, es un producto apto microbiológicamente para el consumo animal y podrá sustituir al menos el $50 \%$ de la proteína (CURY, et al. 2017).

\section{Conclusiones}

Los resultados permitieron demostrar que se puede realizar la separación de cal a temperatura ambiente a condiciones ambientales de $28^{\circ} \mathrm{C}$ que predominan en los procesos de desencalado de pieles. La concentración de agente desencalante (ácido sulfúrico) tuvo un efecto significativo sobre las características evaluadas en los descarnes (\% de grasa, $\mathrm{pH}$ y presencia de cal). Se logró establecer que el tratamiento con mejores resultados para desencalar residuos de descarnes generados en el proceso productivo de curtición fue el de $2 \mathrm{~N}-3 \mathrm{~h}$ debido a que en este se da un ahorro de tiempo y energía en comparación con el tratamiento de $2 \mathrm{~N}-4 \mathrm{~h}$, el cual también tuvo resultados satisfactorios en el desencalado.

Agradecimientos. A la Universidad de Sucre, por el apoyo brindado en esta investigación al permitir utilizar sus instalaciones en el Campus puerta roja. A la Asociación de Curtiembres de Sampués "ACUSAM"

\section{Referencias}

AGUAS, M.Y.; OLIVERO, R.; MERCADO, I.; CURY, K.; MARTINEZ, C.; PARIS, A. 2016. Análisis del tratamiento ideal usando baños termotratados para la separación de cal de los residuos de descarne en curtiembres. Ing.USBmed 7 (1): 20-25.

ALZATE, T.A. 2008 "Proyecto Gestión Ambiental en la industria de Curtiembre en Colombia. Diagnóstico y estrategias"; Disponible: http://www.tecnologiaslimpias.org/Curtiembres/ EstrategiasDiagnostico.pdf.

AOAC. 1990. Official methods of analysis of the association of analytical chemists. 15th Edition. Association of Official Analytical Chemists, Inc. Arlington, Virginia.

ANDRIOLI, E. PETRY, L. GUTTERRES, M. 2015 "Environmentally friendly hide unhairing: Enzymaticoxidative unhairing as an alternative to use of lime and sodium sulfide". Process Safety and Environmental Protection. 93, pp. 9-17.

ARANGO, C. A.; ALZATE, A. M.; TOBÓN, O. L. Manual ambiental sectorial: proyecto gestión ambiental en la industria de curtiembre en Colombia. Medellín: CNPMLTA- Centro Nacional de Producción Más Limpias y Tecnologías Ambientales, 2004, p37. Consultado en Octubre 19 de 2015. "Disponible en: http:// www.tecnologiaslimpias.org/curtiembres/manual.pdf

CORREDOR, R. J. 2006. El residuo líquido de las curtiembres estudio de caso: cuenca alta del rio Bogotá. Revista Ciencia e Ingeniería Neogranadina 16 (2): 14-28

CNPML "CENTRO NACIONAL DE PRODUCCIÓN MÁS LIMPIA". 2004. Proyecto Gestión Ambiental de la industria de curtiembres en Colombia. Manual Ambiental Sectorial. [Online]. Disponible: http://www. cnpml.org.

CURY, K.; AGUAS, Y.; MARTINEZ, A.; OLIVERO, R.; CHAMS, L. 2017. Residuos agroindustriales su impacto, manejo y aprovechamiento. Revista Colombiana de Ciencia Animal. 9 (Supl):122-132. 
LOAYZA, J. E. 2006. Estudio de Investigación: Gestión Integral de Residuos Peligrosos. Boletín electrónico informativo sobre productos y residuos químicos. [Online] 2(13): 1-4. Disponible: http://www.sertox.com.ar.

MANCERA, R.; ÁLVAREZ, R. 2006. Estado del conocimiento de las concentraciones de mercurio y otros metales pesados en peces dulceacuícolas de Colombia. Acta Biológica Colombiana. 11(1): 3-23.

MARTÍNEZ, C.; PARIS, A. 2010. "Determinación del tratamiento óptimo por baños termotratados tipo bombo para la separación de cal de los residuos de descarne del proceso de curtición semiartesanal desarrollado en Sampués-Sucre". Universidad de Sucre, Sincelejo-Colombia.

RAMÍREZ J. A. "Evaluación de la gestión integral de residuos sólidos en las curtiembres de San Benito”. Especialización. Proyecto, Universidad Militar Nueva Granada, Carrera 11 \# 101- 80, Bogotá-Colombia, 2014.

SILVA, S. R.; GONZÁLEZ, M. J. Evaluación técnica del sulfato de calcio anhidro como carga en la fabricación de papeles. Universidad de Chile.1993.

TÉLLEZ, J.; CARVAJAL, R. M.; GAITÁN, A. M. 2004. Aspectos toxicológicos relacionados con la utilización del cromo en el proceso productivo de curtiembres. Revista de la Facultad de Medicina de la Universidad Nacional de Colombia. 52(1):50-61.

VICENTE, F. 2010. "Estudio económico-financiero del aprovechamiento de las grasas extraídas del residuos de descarne unche derivado del proceso de curtición en el Municipio de VillapinzónCundinamarca". [Tesis de Maestría]. Universidad Nacional de Colombia; Bogotá, Colombia. 